\title{
Suporte básico de vida e suporte avançado de vida em cardiologia: proposta de uma nova estratégia para abordagem e prevenção das intercorrências clínicas em cirurgia dermatológica
}

\author{
Basic life support and advanced cardiac life support: proposal of a new strategy
}

to approach and prevent clinical events in dermatologic surgery

\author{
Fábio Roismann Timoner ${ }^{1}$ \\ Manoel Carlos Sampaio de Almeida Ribeiro ${ }^{3}$ \\ Carlos d'Aparecida Santos Machado Filho ${ }^{5}$
}

\author{
Nelson Marcos Ferrari ${ }^{2}$ \\ Frida Liane Plavnik ${ }^{4}$
}

Resumo: Com o aumento da complexidade cirúrgica e comorbidades da população, o risco de intercorrências clínicas em cirurgia dermatológica aumentou nas últimas décadas. Para sua abordagem foi criado um fluxograma baseado na tríade decisória formada pelo estado físico do paciente, grau de sedação e porte do procedimento, indicando o tipo de suporte cardiovascular em cada procedimento. Pacientes submetidos a cirurgias de "porte pequeno" e ASA $\leq 2$ devem receber suporte básico de vida; os submetidos a cirurgias de "porte pequeno" e ASA > 2, a cirurgias de "porte médio" ou a sedação profunda devem receber suporte avançado de vida em cardiologia.

Palavras-chave: Complicações intraoperatórias; Dermatologia; Emergências; Procedimentos cirúrgicos minimamente invasivos; Ressuscitação cardiopulmonar; Suporte vital cardíaco avançado

\begin{abstract}
Since complexity of surgical procedures has increased, and patients' co-morbidities have become more frequent, the risk of clinical complications in dermatologic surgery has also increased in the past decades. In order to better assess these risks, a flowchart based on a ruling triad consisting of the patient's health status, sedation level, and procedure complexity was developed to establish the type of cardiovascular support adequate to each procedure. Patients undergoing small surgeries with ASA $\leq 2$ should be assigned to basic life support; patients undergoing small surgeries with ASA $>2$, and those undergoing medium-sized surgeries or deep sedation should be assigned to advanced cardiac life support (ACLS).

Keywords: Advanced cardiac life support; Cardiopulmonary resuscitation; Dermatology; Emergencies; Intraoperative complications; Surgical procedures, minimally invasive surgical procedures
\end{abstract}

Recebido em 27.10.2008

Aprovado pelo Conselho Consultivo e aceito para publicação em 23.11.09.

* Trabalho realizado na disciplina de dermatologia da Faculdade de Medicina do ABC - São Paulo (SP), Brasil Conflito de interesse: Nenhum / Conflict of interest: None

Suporte financeiro: Nenhum / Financial funding: None

Assistente do setor de cirurgia dermatológica - disciplina de dermatologia - Faculdade de Medicina do ABC - São Paulo (SP), Brasil

Chefe do setor de cirurgia dermatológica - Faculdade de Ciências Médicas da Santa Casa de São Paulo - São Paulo (SP), Brasil.

Assistente da disciplina de medicina social - Faculdade de Ciências Médicas da Santa Casa de São Paulo - São Paulo (SP), Brasil.

Professora afiliada - disciplina de nefrologia - Universidade Federal de São Paulo - São Paulo (SP), Brasil.

Professor regente da disciplina de dermatologia - Faculdade de Medicina do ABC - São Paulo (SP), Brasil.

(C) 2010 by Anais Brasileiros de Dermatologia 
A prática da cirurgia dermatológica é considerada uma intervenção segura, com baixa incidência de complicações, a não ser em alguns relatos que mostram altas taxas de elevação da pressão arterial em procedimentos ambulatoriais sob anestesia local. ${ }^{1,2}$ No entanto, o crescente aumento da complexidade técnica com a lipoaspiração, lifting facial, tumores de maior dimensão e sedação e o envelhecimento da população com suas comorbidades fazem com que os cirurgiões dermatológicos deparem com um maior risco de complicações cirúrgicas e intercorrências clínicas que, embora raras, podem ter alto potencial de mortalidade. ${ }^{3,4}$

Foi desenvolvido um fluxograma (Figura 1) que indica o tipo de suporte clínico a ser seguido em cada cirurgia, suporte básico de vida (SBV) ou suporte avançado de vida em cardiologia (SAVC), em função das características do procedimento e das comorbidades do paciente, aliado a uma rotina de cuidados perioperatórios. No desenvolvimento do fluxograma foram considerados: o estado físico do paciente segundo a classificação da American Society of Anesthesiologists (ASA), o porte cirúrgico dermatológico e o grau de sedação escolhido pela equipe cirúrgica. Esses três itens foram denominados "tríade decisória".

O SBV e o SAVC foram adotados como sistemas padronizados para a abordagem de intercorrências clínicas; o primeiro selecionado para situações consideradas de baixo risco e o último, para situações de maior risco. Para tanto, pelo menos um membro da equipe médica envolvida deve ser qualificado nesses suportes com adequação do ambiente cirúrgico para esse fim.

As cirurgias dermatológicas foram classificadas em "pequeno porte", isto é, de acordo com as cirurgias classicamente consideradas de baixo risco na literatura, ${ }^{5}$ por exemplo, peeling superficial e médio, shaving, curetagem, eletrocoagulação, crioterapia, laser, preenchimento, biópsias, exereses de tumores, cirurgia ungueal, otoplastia, blefaroplastia, lipoaspiração < $100 \mathrm{ml}$ e implante capilar. As cirurgias de "médio porte" são aquelas de risco médio, ${ }^{5}$ como minilifting, lipoaspiração $>100 \mathrm{ml}$, peeling de Baker e tumores extensos. Sedação foi definida como o grau de sedação capaz de alterar os reflexos das vias aéreas superiores e produzir alterações hemodinâmicas.

Foi elaborada também uma estratégia complementar que inclui os pré-requisitos da equipe e ambiente cirúrgico, avaliação pré-operatória e cuidados perioperatórios (Quadro 1).

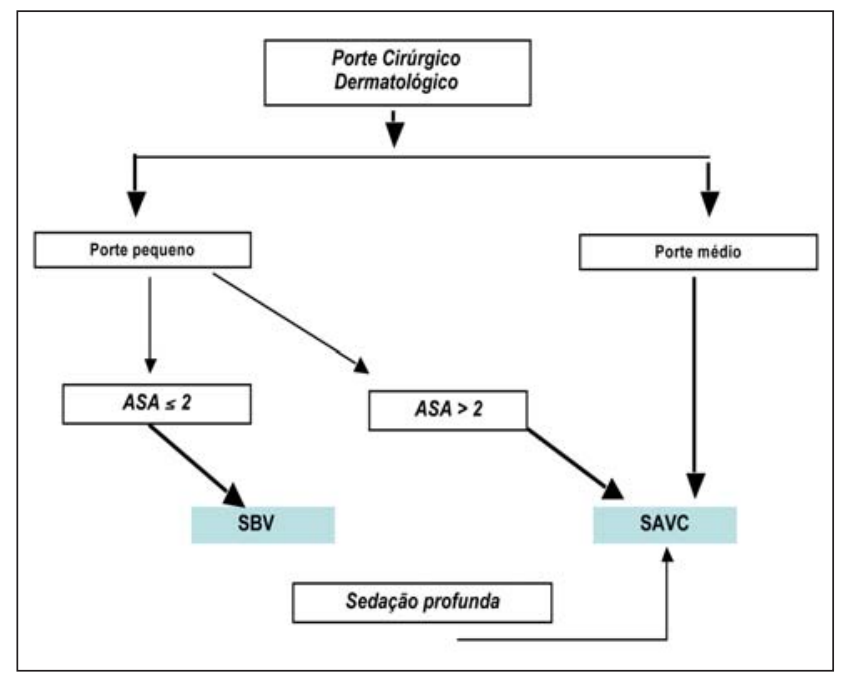

FigurA 1: Fluxograma para a abordagem de intercorrências clínicas em cirurgia dermatológica

O fluxograma resultou nas seguintes situações: 1- cirurgia de pequeno porte, ASA 1 ou 2, indicativa de SBV; 2- cirurgia de pequeno porte, ASA $>2$, indicativa de SAVC; e 3- cirurgia de médio porte ou sedação profunda, indicativas de SAVC.

Este é o primeiro estudo na literatura a propor uma estratégia diferenciada para os procedimentos dermatológicos, com base na tríade: estado físico do paciente, porte cirúrgico dermatológico e grau de sedação.

Fader e Johnson abordaram as complicações que podem ocorrer no consultório dermatológico na rotina, mencionando a necessidade de treinamento dos profissionais envolvidos nos suportes básicos ou avançados de vida em cardiologia (SBV ou SAVC). As observações foram direcionadas ao tempo que a equipe apropriada levaria para chegar ao local e não ao porte do procedimento ou estado físico do paciente. ${ }^{4}$

Embora a maioria dos procedimentos dermatológicos seja considerada de baixo risco (inferior a 1\%), a lipoaspiração, a exerese de tumores extensos e o lifting facial se enquadram no risco médio, com taxas de complicações variando de 1 a 5\%. ${ }^{4}$ O peeling de fenol, sob a fórmula de Baker, apresenta risco direto de toxicidade cardíaca, causando arritmias que podem ser fatais, além de toxicidade renal, que eleva o risco em pacientes cardiopatas ou nefropatas. ${ }^{6} \mathrm{Um}$ fato importante associado às intercorrências clínicas é a elevação nos níveis de catecolaminas, que chegam a atingir valores 40 vezes acima do valor basal e são decorrentes de 
QUADRo 1: Estratégia complementar para prevenção de intercorrências em cirurgia dermatológica

\begin{tabular}{|c|c|}
\hline \multicolumn{2}{|r|}{ A - Pré-requisitos dos profissionais envolvidos: } \\
\hline • & Treinamento supervisionado em cirurgia dermatológica \\
\hline - & Treinamento em SBV ou SAVC \\
\hline$\bullet$ & Atuação em ambiente adaptado ao SBV ou SAVC \\
\hline \multirow[t]{4}{*}{$\bullet$} & Sistema médico de emergência (SME) \\
\hline & - Plano para providências imediatas da equipe em caso de intercorrências médicas \\
\hline & Disponibilidade imediata de telefones das centrais de ambulâncias UTI com desfibrilador \\
\hline & Escala de brigadas de emergências \\
\hline \multicolumn{2}{|c|}{ B - Avaliação pré-operatória: } \\
\hline • & $\begin{array}{l}\text { Otimização prévia dos medicamentos que possam ter influência no procedimento cirúrgico (interações } \\
\text { medicamentosas entre antiagregantes plaquetários, anticoagulantes, anti-hipertensivos, hipoglicemiantes e } \\
\text { anestésico) }\end{array}$ \\
\hline • & Determinação do estado físico de acordo com os critérios da ASA e otimização das patologias não compensadas \\
\hline - & Classificação do porte cirúrgico dermatológico \\
\hline$\bullet$ & Decisão da sedação \\
\hline$\bullet$ & Orientações por escrito dos cuidados perioperatórios \\
\hline • & Aplicação do fluxograma \\
\hline \multicolumn{2}{|c|}{ C - Ato cirúrgico: } \\
\hline$\bullet$ & $\begin{array}{l}\text { Avaliação da enfermagem: dados vitais, sintomas e sinais de urgências ou emergências, informação sobre } \\
\text { medicamentos contraindicados e específicos para as comorbidades }\end{array}$ \\
\hline$\bullet$ & Rotinas de monitoração para diabéticos e hipertensos \\
\hline$\bullet$ & Ansiolíticos \\
\hline$\bullet$ & Músicas relaxantes \\
\hline • & Avaliação dos dados vitais antes da alta \\
\hline • & Reforço das orientações pós-operatórias \\
\hline
\end{tabular}

diferentes níveis de estresse e potencial de geração de dor, complexidade técnica e duração do procedimento.?

Salienta-se que as baixas taxas de riscos referentes às complicações em cirurgia dermatológica mencionam as complicações intraoperatórias, mas não nas 48 horas do pós-operatório. Estima-se que mais da metade dos óbitos que ocorrem no período pós-operatório sejam devidos a eventos cardíacos, e nos pacientes coronariopatas a isquemia é frequente no pós-operatório. Por vezes, a ativação do sistema nervoso simpático e da medula adrenal, seja pela dor ou por distúrbios homeostáticos, pode acarretar alterações da frequência cardíaca e trabalho cardíaco, o que poderá agudizar o quadro. ${ }^{8}$

Ainda outros fatores de risco se integram aos procedimentos mais complexos, como a quantidade de anestésico, topografia cirúrgica, tempo cirúrgico, área de descolamento, sangramento, os quais podem contribuir para essas ocorrências."

A inclusão do estado físico do paciente baseado na classificação da ASA deve-se à sua praticidade e ampla aceitação, e também por ser de maior valor preditivo para complicações fatais, sobretudo em pacientes com ASA > 2, principalmente cardiológicas, superando os riscos do próprio procedimento e o grau de sedação. ${ }^{10}$

A adoção da SBV e SAVC permite uma atuação rápida e coordenada. Essa estratégia pode também ser projetada para a escolha de cirurgias que poderão ser feitas de forma segura em ambiente de consultório, por ter um perfil mais aproximado de SBV, ou em centros cirúrgicos ambulatoriais ou hospitalares, que se aproximam do SACV.

Em resumo, a aplicabilidade desta proposta de avaliação e adequação dos pacientes na cirurgia dermatológica será otimizada com a utilização deste fluxograma, visando ao melhor suporte individualizado para cada situação. Para tanto, estudos prospectivos posteriores poderão confirmar sua relevância e talvez permitir seu aperfeiçoamento e redimensionamento. 


\section{REFERÊNCIAS}

1. Pimentel ERA, Oliveira JP, Bloch LD, Niwa ABM. Risco de complicações durante a cirurgia dermatológica: protocolo das exéreses em fuso. An Bras Dermatol. 2005;80:493-8.

2. Larson MJ, Taylor RS. Monitoring vital signs during outpatient Mohs and post-Mohs reconstructive surgery performed under local anesthesia. Dermatol Surg. 2004;30:777-83.

3. Fader DJ, Johnson TM. Medical issues and emergencies in the dermatology office. J Am Acad Dermatol. 1997;36:1-16.

4. Eagle KA, Brundage BH, Chaitman BR, Ewy GA, Fleisher LA, Hertzer NR, et al. Guidelines for perioperative cardiovascular evaluation for noncardiac surgery. Report of the American College of Cardiology/American Heart Association Task Force on Practice Guidelines. Committee on Perioperative Cardiovascular Evaluation for Noncardiac Surgery. Circulation. 1996;93:1278-317.

5. Dias DL, Bittencourt LAK, Cavicchio JR, Figueiredo MJ, Simões AM. Importância e fundamentos da avaliação pré-operatória para pacientes submetidos a cirurgia não cardíaca: quem deve ser avaliado e quem deve avaliar. Rev Soc Cardiol Estado de São Paulo. 2000; 10:259-67.

6. Landau M. Cardiac complications in deep chemical peels. Dermatol Surg. 2007;33:190-3.
7. Malamed SF. Medical emergencies in the dental office. 4th ed. St Louis: Mosby-Year Book; 1993. p.229-53.

8. Baruzzi ACA, Knobel E. Quais pacientes devem receber cuidados de UTI no pós operatório e que condutas devem ser tomadas para prevenir complicações cardiovasculares? Rev Soc Cardiol Estado de São Paulo. 2000;10:282-8.

9. Yoho RA, Romaine JJ, O'Neil D. Review of liposuction, abdominoplasty and face-lift mortality and morbidity risk literature. Dermatol Surg. 2005;31:733-43.

10. Nicolai JN, Maia, LN, Costa OMC. Abordagem pré-operatória do paciente com síndrome isquêmica aguda recente. Rev Soc Cardiol Estado de São Paulo. 2000;10:324-33

ENDEREÇO PARA CORRESPONDÊNCIA / MAILING ADDRESS:

Fábio R. Timoner

Av. Índico, 499 -Jardim do Mar -

09750601 São Bernardo do Campo, SP.

Tel./fax: $117872-200411$ 4123-5805.

E-mail: drfabiotimoner@botmail.com

Como citar este artigo/How to cite this article: Timoner FR, Ferrari NM, Ribeiro MCSA, Plavnik FL, Machado Filho CAS. Suporte básico de vida e suporte avançado de vida em cardiologia: proposta de uma nova estratégia para abordagem e prevenção das intercorrências clínicas em cirurgia dermatológica. An Bras Dermatol. 2010;85(1):115-8. 